\title{
The Dual Solutions and Stability Analysis of Nanofluid Flow using Tiwari-Das Modelover a Permeable Exponentially Shrinking Surface with Partial Slip Conditions
}

\author{
${ }^{1,2}$ Sumera Dero, ${ }^{1}$ Azizah Mohd Rohni and ${ }^{1}$ Azizan Saaban \\ ${ }^{1}$ School of Quantitative Sciences, University of Utara Malaysia, 06010 Sintok, Kedah, Malaysia \\ ${ }^{2}$ University of Sindh, Jamshoro, Pakistan
}

\begin{abstract}
The main object of present research is to find the multiple solutions of the nanofluid flow of two-dimensional water-based nanofluid over the exponentially shrinking surface in existence of slip conditions and the thermal radiation. The governing equations are transformed into the coupled nonlinear similarity equations by exponential type of the similarity transformations. The obtained equations have been solved numerically to get the velocities and the temperature distribution profiles in respective of the boundary layer flow. From the numerical solutions, it is observed that there exist the dual solutions for the certain considered parameters. The results are presented to demonstrate the effect of different parameters consisting the velocity and thermal slip, the thermal radiation parameter and the volumetric fractions of the three different kinds of the solid nanoparticles, like as, Copper $(\mathrm{Cu})$, silver $(\mathrm{Ag})$ and aluminium by means of water as the base fluid. In last, the stability analysis is performed in which it is shown that the first solution is stable and physically possible.
\end{abstract}

Key words: Tiwari and Dass Model, slip conditions, thermal radiation, dual solution, stability analysis, parameters

\section{INTRODUCTION}

In fluid flow, the existence of the no-slip condition is assumed at the solid boundary where the velocity is zero because of the resistance of the solid surface of the boundary. Whereas in many engineering applications, the no-slip conditions do not hold always in realism (Shu et al., 2016). Maxwell (1879) examined the viscous stresses rising in the rarefied gases. On this, the reviewer suggested that it would be more beneficial if the Maxwell might be used the theoretical results to derive the velocity boundary conditions to the rarefied gases flows adjacent to the solid surfaces. Therefore, Maxwell presented the now-famous velocity slip boundary condition theory. It is observed that in many industrial and engineering processes, the effects of the slip rise along the solid wall of the pipes or surfaces. A common approach in the study of the slip occurrences is Navier velocity slip conditions. The thermal slip conditions as well as concentration slip conditions, also, rise in the different industrial and engineering processes. It is seen that the boundary layer slip flow problems arise during the polish of the artificial hearts valves and interior cavities. Many researchers considered the no-slip effect such as Hamid and Khan (2018), Hayat et al. (2018a, b),
Hamid et al. (2018), Kumaran and Sandeep (2017) and Ramzan et al. (2017). The object of this paper is also to study the velocity as well as thermal slip effect in nanofluid flow on the exponential shrinking sheet.

In updated manufacturing and thermal processes, the working fluids have got great importance due to decreasing or increasing energy release in the systems whereas it's their efficacies, especially depends upon the heat capacity, thermal conductivity and the physical qualities. The thermal conductivity parameter plays a vital role to control the heat transfer performance of the systems. Whereas thermal conductivity, especially of the metals is significantly greater as compared to the common base fluids, the main development which occurred in this field is the suspension of the metallic solid smallest in the base fluid that much increased the thermal conductivity of working fluid. That new method by means of nano-sized solid particles was introduced by Choi and Eastman (1995) that fluids are named as the nanofluids. The efficiency of the thermal transfer improvement is found due to the presence of the different factors such as the number of the suspended nanoparticles particles, particle shape and material type. The nanoparticles are formed from the various materials, like as metal nitrides ( $\mathrm{SiN}$, $\mathrm{AlN})$, oxide ceramics $\left(\mathrm{CuO}, \mathrm{Al}_{2} \mathrm{O}_{3}\right)$, metals $(\mathrm{Au}, \mathrm{Ag}, \mathrm{Cu})$,

Corresponding Author: Sumera Dero, University of Sindh, Jamshoro, Pakistan 
carbide ceramics (Tic, Sic), carbons (e.g., graphite, fluorine, carbon nanotubes, diamond) and the working nano-particles. Since, last many years, various experimental studies on thermal conductivity related to the nanofluids have been considered. The experimental studies have exposed that the 1-5\% volume of the metallic oxide or solid metallic particles increases the $20 \%$ thermal conductivity of the mixture as compared to common base fluid reported by Eastman (1999), Xuan and Roetzel (2000) and Masuda et al. (1993). The phenomena of the nanofluid through stretching surface for the boundary layer laminar flow was first done by Khan and Pop (2010). The new improvements in this field, the vast quantity of literature concerned to the nanofluid for newtonian as well as non-newtonian models are available but we have referred few studies (Kashyap and Dass; 2018; Khan et al., 2018; Emami et al., 2018; Rashad et al., 2017; Sandeep and Reddy, 2017; Reddy et al., 2017; Sulochana and Sandeep, 2016; Hayat et al., 2016; Raza et al., 2016). Bachok et al. (2012) considered the stagnation point flow on the exponential shrinking/ stretching surface. Ismail et al. (2016) examined the stagnation point flow on the exponentially shrinking surface and performed stability. Bhattacharyya and Vajravelu (2012) studied stagnation point flow through exponential shrinking surface and found dual solutions but did not perform stability.

In views of the papers by Bachok et al. (2012), Ismail et al. (2016) and Bhattacharyya and Vajravelu (2012), this study is concerned with problems of the steady nanofluid flows and the heat transfer through the exponential shrinking surface in presence of the thermal radiation and hydrodynamic and thermal slip conditions. The researchers have introduced the many mathematical models concerned to the nanofluids as to compare to experimental data with the theory observations. Yet, there still much needs of the further, investigations to develop a comprehensive theory for the prediction of the thermal conductivity in the nanofluids. But there happen few empirical correlations to compute thermal conductivity of the two-phase combinations. A mathematical model of the nanofluid introduced by Buongiorno (2006) has been recently applied by the many researchers, like as Nield and Kuznetsov $(2009,2011)$. The different from the Buongiorno and Nield and Kuznetsov Models there are also other simpler mathematical models of the nanofluids introduced by Khanafer et al. (2003), Tiwari and Das (2007) that investigate behaviors of the nanofluids by considering the solid volumetric fractions. In this study, the model of Tiwari and Das (2007) is used that is also used by many researchers previously such as Sheremet et al. (2016), Aghamajidi et al. (2018) and Mustafa et al. (2018).
In present research, we have extended the idea of Bachok et al. (2012) to examine the two-dimensional flow of the nanofluid on the exponentially shrinking surface. In this study, there has been taken water as a base fluid and also considered the effect of volume fraction of the solid nano-particle are considered. Further, there has been taken three different kinds of the solid nanoparticles like the Copper $(\mathrm{Cu})$, silver $\left(\mathrm{Al}_{2} \mathrm{O}_{3}\right)$ and aluminium. By applying similarity transformation, boundary layer momentum and the energy equations are reduced to the system of ordinary differential equations. The numerical solutions of the reduced differential equations have been obtained by applying the shooting technique with shootlib function in the Maple Software. We performed stability in order to show the first solution is always stable with the help of bvp $4 \mathrm{c}$ solver. The graphical results of different applied parameters are defined by graphs with the discussion. The physical behaviors of the local Nusselt as well as Sherwood number have been discussed by the graphs.

\section{MATERIALS AND METHODS}

Mathematical formulation: There has been considered the incompressible laminar two-dimensional boundary layer steady state nanofluid flow and the heat transfer with thermal radiation on the exponentially stretching and shrinking surface (Fig. 1) with slip conditions. The governing conservation (continuity), momentum and the energy equations are written as:

$$
\begin{gathered}
\frac{\partial \mathrm{u}}{\partial \mathrm{x}}+\frac{\partial v}{\partial \mathrm{y}}=0 \\
\mathrm{u} \frac{\partial \mathrm{u}}{\partial \mathrm{x}}+v \frac{\partial \mathrm{u}}{\partial \mathrm{y}}=\frac{\mu_{\mathrm{nf}}}{\rho_{\mathrm{nf}}} \frac{\partial^{2} \mathrm{u}}{\partial \mathrm{y}^{2}} \\
\mathrm{u} \frac{\partial \mathrm{T}}{\partial \mathrm{x}}+v \frac{\partial \mathrm{T}}{\partial \mathrm{y}}=\alpha_{\mathrm{nf}} \frac{\partial^{2} \mathrm{~T}}{\partial \mathrm{y}^{2}}-\frac{1}{\left(\rho \mathrm{c}_{\mathrm{p}}\right)_{\mathrm{nf}}} \frac{\partial \mathrm{q}_{\mathrm{r}}}{\partial \mathrm{y}}
\end{gathered}
$$

The boundary conditions are:

$$
\begin{aligned}
& v=v_{\mathrm{w}}(\mathrm{x}), \mathrm{u}=\mathrm{u}_{\mathrm{w}}+\mathrm{N}_{\mathrm{s}} \vartheta \frac{\partial \mathrm{u}}{\partial \mathrm{y}}, \mathrm{T}=\mathrm{T}_{\mathrm{w}}(\mathrm{x})+ \\
& \mathrm{K} \frac{\partial \mathrm{T}}{\partial \mathrm{y}} \text { as } \mathrm{y} \rightarrow 0 \mathrm{u} \rightarrow 0, \mathrm{~T} \rightarrow \mathrm{T}_{\infty} \text { as } \mathrm{y} \rightarrow \infty
\end{aligned}
$$

Here, $u$ and $v$ are velocity components in directions of $x$-axis and $y$-axis, respectively $N_{s}=N_{1} e^{-x / 21}$ is the velocity slip and $\mathrm{N}_{1}$ is the initial values of velocity factor, 


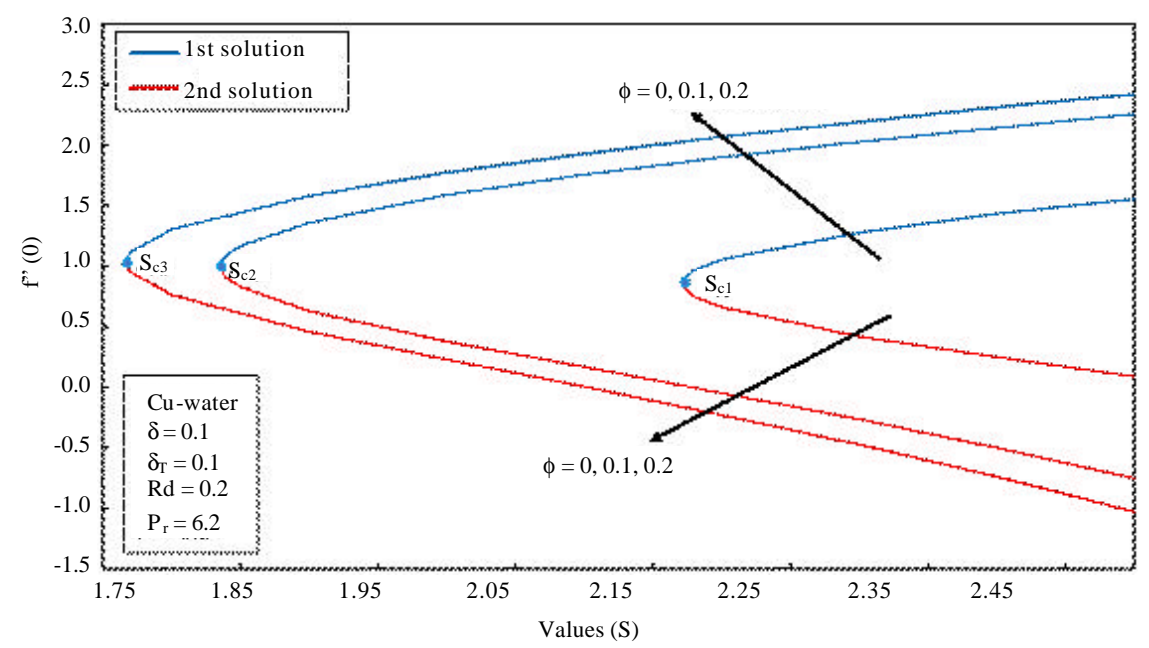

Fig. 1: The variation of $\mathrm{f}^{\prime \prime}(0)$ along $\delta$ at different values of $\phi(0 \leq \phi \leq 0.2)$ for the Cu-water operational fluid

$\mathrm{T}$ denotes the temperature at boundary layer, $\mathrm{T}_{\mathrm{w}}(\mathrm{x})$ stands for the variable temperature of the surface and $T_{w}(x)=$ $\mathrm{T}_{\infty}+\mathrm{T}_{0} \mathrm{e}^{\mathrm{z} / 21}, \mathrm{~T}_{\infty}$ free stream Temperature, $\mathrm{T}_{0}$ is the constant that measures the rate of the increase in temperature with surface, $\mathrm{K}=\mathrm{K}_{1} \mathrm{e}^{-\mathrm{x} / 21}$ denotes the thermal slip and $\mathrm{K}_{1}$ initial value of the thermal factor, $\mu_{\mathrm{nf}}$ stands for viscosity of nanofluid, $\alpha_{\mathrm{nf}}$ denotes thermal diffusivity of the nanofluid and $\rho_{\text {nf }}$ density of the nanofluid, $\mathrm{q}_{\mathrm{r}}=-4 \sigma^{*} / \mathrm{k}^{*} \partial \mathrm{T}^{4} / \partial \mathrm{y}$ indicates radiative fluxthat are presented as:

$$
\begin{aligned}
& \rho_{\mathrm{nf}}=(1-\phi) \rho_{\mathrm{f}}+\phi \rho_{\mathrm{s}} ;\left(\rho \mathrm{c}_{\mathrm{p}}\right)_{\mathrm{nf}}=(1-\phi)\left(\rho \mathrm{c}_{\mathrm{p}}\right)_{\mathrm{f}}+\phi\left(\rho \mathrm{c}_{\mathrm{p}}\right)_{\mathrm{s}} \\
& \mathrm{a}_{\mathrm{nf}}=\frac{\mathrm{k}_{\mathrm{nf}}}{\left(\rho \mathrm{c}_{\mathrm{p}}\right)_{\mathrm{nf}}} \frac{\mathrm{k}_{\mathrm{nf}}}{\mathrm{k}_{\mathrm{f}}}=\frac{\left(\mathrm{k}_{\mathrm{s}}+2 \mathrm{k}_{\mathrm{f}}\right)-2 \phi\left(\mathrm{k}_{\mathrm{f}}-\mathrm{k}_{\mathrm{s}}\right)}{\left(\mathrm{k}_{\mathrm{s}}+2 \mathrm{k}_{\mathrm{f}}\right)+\phi\left(\mathrm{k}_{\mathrm{f}}-\mathrm{k}_{\mathrm{s}}\right)} ; \mu_{\mathrm{nf}}=\frac{\mu_{\mathrm{f}}}{(1-\phi)^{2.5}}
\end{aligned}
$$

Here, $\phi$ is volumetric fraction of solid nanoparticles $\left(\rho_{\mathrm{cp}}\right)_{\mathrm{nf}}$ indicates heat capacity of the nanofluid, $k_{n f}$ denotes thermal conductivity of the nanofluid, $\mathrm{k}_{\mathrm{s}}$ and $\mathrm{k}_{\mathrm{f}}$ are the thermal conductivities concerned to solid volume fraction and base fluid, respectively whereas $p_{s}$ and $p_{f}$ are densities related to the nano-particles volume fraction and the base fluid, respectively. It might be noted that, here, the $k_{n f}$ stands for spherical nano-particles, already mentioned by Sulochana and Sandeep (2016). The $\mu_{\mathrm{nf}}$ that indicates the viscosity of nanofluid is already guessed by Brinkman and the $\mu_{\mathrm{f}}$ viscosity of the base fluid with dilute mixture of spherical shaped nano-sized particles. The shrinking $\mathrm{u}_{\mathrm{w}}$ is defined by:

$$
\mathrm{u}_{\mathrm{w}}=-\mathrm{U}_{\mathrm{w}} \mathrm{e}^{\mathrm{z} / 1}
$$

The governing Eq. 1-3 subjected to boundary conditions Eq. 4 are reduced by applying the following similarity transformation:

$$
\psi=\sqrt{2 \vartheta_{\mathrm{f}} 1 \mathrm{U}_{\mathrm{w}}} \mathrm{e}^{z / 21} \mathrm{f}(\eta) ; \theta(\eta)=\frac{\mathrm{T}-\mathrm{T}_{\infty}}{\mathrm{T}_{\mathrm{w}}-\mathrm{T}_{\infty}} ; \eta=\mathrm{y} \sqrt{\frac{\mathrm{U}_{\mathrm{w}}}{2 \vartheta_{\mathrm{f}} 1}} \mathrm{e}^{\mathrm{z} / 21}(7)
$$

where, $\eta$ denotes the similarity variable, $\vartheta_{\mathrm{f}}$ denotes kinematic viscosity concerned to thebase fluid and the $\psi$ indicates stream function explained as $\mathrm{u}=\partial \psi / \partial \mathrm{y}$ and $v=-\partial \psi / \partial \mathrm{x}$ that satisfy in Eq. 1. Using similarity, variables (Eq. 2, 3 and 7) are reduced to the following non-linear ordinary differential equations:

$$
\begin{gathered}
\frac{1}{(1-\phi)^{2.5}\left((1-\phi)+\phi\left(\rho_{s} / \rho_{\mathrm{f}}\right)\right)} \mathrm{f}^{\prime \prime \prime}+\mathrm{f}^{\prime \prime} \mathrm{f}-2(\mathrm{f})^{2}=0 \\
\frac{\mathrm{k}_{\mathrm{nf}} / \mathrm{k}_{\mathrm{f}}}{\mathrm{P}_{\mathrm{r}}\left((1-\phi)+\phi \frac{\left(\rho c_{\mathrm{p}}\right)_{\mathrm{s}}}{\left(\rho c_{\mathrm{p}}\right)_{\mathrm{f}}}\right.}\left(1+\frac{4}{3} \mathrm{Rd}\right) \theta^{\prime \prime}+\theta^{\prime} \mathrm{f}-\theta \mathrm{f}^{\prime}=0
\end{gathered}
$$

Subjecting to boundary conditions (Eq. 4) that becomes:

$$
\begin{aligned}
& f(0)=S ; f^{\prime}(0)=-1+\delta f^{\prime \prime}(0) ; \theta(0)=1+\delta_{\mathrm{T}} \theta^{\prime}(0) \\
& f^{\prime}(\eta) \rightarrow 0 ; \theta(\eta) \rightarrow 0 ; \varnothing(\eta) \rightarrow 0 \text { as } \eta \rightarrow \infty
\end{aligned}
$$

where, primes stand for derivatives with respect to $\eta$, $\mathrm{Rd}=4 \sigma^{*} \mathrm{~T}^{3}{ }_{\mathrm{o}} / \mathrm{k}^{*} \mathrm{k}_{\mathrm{nf}}$ is thermal radiation and $\mathrm{P}_{\mathrm{r}}=\vartheta_{\mathrm{f}} / \alpha_{\mathrm{f}}$ is the Prandtl number.

The important physical factors are skin friction Coefficient $\mathrm{C}_{\mathrm{f}}$ and local Nusselt number $\mathrm{Nu}_{\mathrm{z}}$ that are explained as: 


$$
\mathrm{C}_{\mathrm{f}}=\frac{\mu_{\mathrm{nf}}}{\rho_{\mathrm{f}} \mathrm{u}_{\mathrm{w}}^{2}}\left(\frac{\partial \mathrm{u}}{\partial \mathrm{y}}\right)\left|\mathrm{y}=0, \mathrm{Nu}_{\mathrm{z}}=-\frac{\mathrm{xk}_{\mathrm{nf}}}{\mathrm{k}_{\mathrm{f}}\left(\mathrm{T}_{\mathrm{w}}-\mathrm{T}_{\infty}\right)}\left(\frac{\partial \mathrm{T}}{\partial \mathrm{y}}\right)\right| \mathrm{y}=0
$$

Where:

$\mathrm{k}_{\mathrm{nf}}=$ Thermal conductivity of nanofluids

$\mu_{\mathrm{nf}}=$ Dynamic viscosity

Applying similarity variables (Eq. 7), we have obtained:

$$
\sqrt{\operatorname{Re}} C_{\mathrm{f}}=\frac{1}{(1-\phi)^{2.5}} \mathrm{f}^{\prime \prime}(0) ; \sqrt{\frac{1}{\operatorname{Re}}} \mathrm{Nu}_{\mathrm{z}}=-\frac{\mathrm{k}_{\mathrm{nf}}}{\mathrm{k}_{\mathrm{f}}}\left(1+\frac{4}{3} \mathrm{Rd}\right) \theta^{\prime}(0)
$$

Stability analysis: To perform the stability analysis, there has been considered unsteady problems. Equation 1 holds automatically whereas (Eq. 2) and (3) are rewritten as:

$$
\begin{gathered}
\frac{\partial u}{\partial t}+u \frac{\partial u}{\partial x}+v \frac{\partial u}{\partial y}=\frac{\mu_{n f} \partial^{2} u}{\rho_{n f} \partial y^{2}} \\
\frac{\partial T}{\partial t}+u \frac{\partial T}{\partial x}+v \frac{\partial T}{\partial y}=\alpha_{n f} \frac{\partial^{2} T}{\partial y^{2}}-\frac{1}{\left(\rho c_{p}\right)_{n f}} \frac{\partial \mathrm{q}_{\mathrm{r}}}{\partial y}
\end{gathered}
$$

Here, $t$ indicates time using the variables (Eq. 7), there has been introducednon-dimensional variables as:

$$
\begin{aligned}
& \psi=\sqrt{2 \vartheta 1 \mathrm{U}_{\mathrm{w}}} \mathrm{e}^{\mathrm{z} / 2 \mathrm{f}} \mathrm{f}(\eta, \tau) ; \eta=\mathrm{y} \sqrt{\frac{\mathrm{U}_{\mathrm{w}}}{2 \vartheta 1}} \mathrm{e}^{\mathrm{z} / 21} \\
& \tau=\frac{\mathrm{U}_{\mathrm{w}}}{21} \mathrm{e}^{\mathrm{z} / 1} \mathrm{t} ; \theta(\eta, \tau)=\left(\mathrm{T}-\mathrm{T}_{\infty}\right) /\left(\mathrm{T}_{\mathrm{w}}-\mathrm{T}_{\infty}\right)
\end{aligned}
$$

Equation 13 and 14 are written as:

$$
\begin{gathered}
\frac{1}{(1-\phi)^{2.5}\left((1-\phi)+\phi\left(\rho_{\mathrm{s}} / \rho_{\mathrm{f}}\right)\right)} \frac{\partial^{3} \mathrm{f}(\eta, \tau)}{\partial \eta^{3}}+\frac{\partial^{2} \mathrm{f}(\eta, \tau)}{\partial \eta^{2}} \\
\mathrm{f}(\eta, \tau)-2\left(\frac{\partial \mathrm{f}(\eta, \tau)}{\partial \eta}\right)^{2}-\frac{\partial^{2} \mathrm{f}(\eta, \tau)}{\partial \mathrm{t} \partial \eta}=0 \\
\frac{\mathrm{k}_{\mathrm{nf}} / \mathrm{k}_{\mathrm{f}}}{\mathrm{P}_{\mathrm{r}}\left((1-\phi)+\phi \frac{\left.\left(\rho c_{\mathrm{p}}\right)_{\mathrm{s}}\right)}{\left(\rho c_{\mathrm{p}}\right)_{\mathrm{f}}}\right)}\left(1+\frac{4}{3} \mathrm{Rd}\right) \frac{\partial^{2} \theta(\eta, \tau)}{\partial \eta^{2}}+\mathrm{f}(\eta, \tau) \\
\frac{\partial \theta(\eta, \tau)}{\partial \eta}-\frac{\partial \mathrm{f}(\eta, \tau)}{\partial \eta} \theta(\eta, \tau)-\frac{\partial \theta(\eta, \tau)}{\partial \tau}=0
\end{gathered}
$$

Subjected to boundary conditions:

$$
\begin{gathered}
f(0, \tau)=S, \frac{\partial f}{\partial \eta}(0, \tau)=-1+\delta \frac{\partial^{2} f}{\partial \eta^{2}}, \theta(0, \tau)=1+\delta_{T} \frac{\partial \theta(0, \tau)}{\partial \eta} \\
f^{\prime}(\eta, \tau) \rightarrow 0, \theta(\eta, \tau) \rightarrow 0 \text { as } \eta \rightarrow \infty
\end{gathered}
$$

To perform the stability of steady state flow solutions $f(\eta)=f_{0}(\eta)$ and $\theta(\eta)=\theta_{0}(\eta)$ that satisfy the problems (Eq. 1-4), we may write (Rosca and Pop, 2013; Weidman et al., 2006):

$$
\begin{aligned}
& f(\eta, \tau)=f_{0}(\eta)+e^{-\varepsilon \tau} F(\eta, \tau) \\
& \theta(\eta, \tau)=\theta_{0}(\eta)+e^{-\varepsilon \tau} G(\eta, \tau)
\end{aligned}
$$

Here, $G(\eta, \tau)$ and $F(\eta, \tau)$ and are the small concerned to $\theta_{0}(\eta)$ and $f_{0}(\eta)$ and $\varepsilon$ is the unknown eigenvalue and the solutions of eigenvalues problems (Eq. 16-18) provide the unlimited set of the eigenvalues $\boldsymbol{\varepsilon}_{1}<\boldsymbol{\varepsilon}_{2}<\boldsymbol{\varepsilon}_{3} \ldots$, if smallest eigenvalue is positive then there occurs initial decay and flow is stable whereas in cases of negative there occurs initial growth of the disturbances and the flow is called unstable. Using Eq. 19 in 16 and 17. The solutions $f(\eta)=f_{0}(\eta)$ and $\theta(\eta)=\theta_{0}(\eta)$ of steady state Eq. 8 and 9 are found by setting $\tau=0$. Due to that, there will be solved following linear eigenvalues problems:

$$
\begin{gathered}
\frac{1}{(1-\phi)^{2.5}\left((1-\phi)+\phi\left(\rho_{\mathrm{s}} / \rho_{\mathrm{f}}\right)\right)} \mathrm{F}_{0}^{\prime \prime}+\mathrm{f}_{0} \mathrm{~F}_{0}^{\prime \prime}+\mathrm{F}_{0} \mathrm{f}_{0}^{\prime \prime}-4 \mathrm{f}_{0}^{\prime} \mathrm{F}_{0}^{\prime}+\varepsilon \mathrm{F}_{0}^{\prime}=0 \\
\frac{\mathrm{k}_{\mathrm{nf}} / \mathrm{k}_{\mathrm{f}}}{\mathrm{P}_{\mathrm{r}}\left((1-\phi)+\phi \frac{\left(\rho \mathrm{c}_{\mathrm{p}}\right)_{\mathrm{s}}}{\left(\rho \mathrm{c}_{\mathrm{p}}\right)_{\mathrm{f}}}\right)}\left(1+\frac{4}{3} \mathrm{Rd}\right) \mathrm{G}_{0}^{\prime \prime}+\mathrm{f}_{0} \mathrm{G}_{0}^{\prime}+ \\
\mathrm{F}_{0} \theta_{0}^{\prime}-\mathrm{f}_{0}^{\prime} \mathrm{G}_{0}-\mathrm{F}_{0}^{\prime} \theta_{0}+\varepsilon \mathrm{G}_{0}=0
\end{gathered}
$$

Subjected to boundary conditions:

$$
\begin{aligned}
& \mathrm{F}_{0}(0)=0, \mathrm{~F}_{0}^{\prime}(0)=\delta \mathrm{F}_{0}^{\prime \prime}(0), \mathrm{G}_{0}(0)=\delta_{\mathrm{T}} \mathrm{G}_{0}^{\prime}(0) \\
& \mathrm{F}_{0}^{\prime}(\eta) \rightarrow 0, \mathrm{G}_{0}(\eta) \rightarrow 0 \text { as } \eta \rightarrow \infty
\end{aligned}
$$

It may be noted that at certain values of the of some parameters are $\mathrm{P}_{\mathrm{r}}=6.2, \phi=0.1$ and $\mathrm{Rd}=0.2$, the stability to concerned steady flow solutions $f_{0}(\eta)$ and $\theta_{0}(\eta)$ are found at minimum eigenvalues that has been proposed by Harris et al. (2009), the limit of the probable eigenvalues are found with relaxing the boundary condition at $\mathrm{G}_{0}(\eta)$ and $F_{0}{ }^{\prime}(\eta)$. In this problem, there has been relaxed the condition at $\mathrm{F}_{0}{ }^{\prime}(\eta) \rightarrow 0$ as $\eta \rightarrow \infty$ and at constant value of the $\mathrm{P}_{\mathrm{r}}=6.2, \phi=0.1$ and $\mathrm{Rd}=0.2$, we have solved the system of Eq. $20-22$ with the boundary condition $\mathrm{F}_{0}{ }^{\prime \prime}(0)=1$. 


\section{RESULTS AND DISCUSSION}

In this study, there has been considered nanofluids made up of three kinds of the solid nanoparticles that are Copper $(\mathrm{Cu})$, silver $(\mathrm{Ag})$ and aluminium with water as a based fluid. The effects of the solid volume fractions $\phi$ are examined in a range of $0 \leq \phi \leq 0.2$ with Prandtl number 6.2 that value is concerned to water which bases fluid of the present research. Hayat et al. $(2018 \mathrm{a}, \mathrm{b})$ and Ahmad et al. (2011) have described the thermo-physical properties of $\mathrm{Cu}, \mathrm{Ag}$ and aluminium and the base fluid water that is shown in Table 1. In case of the without slip conditions and in absence of shrinking effect, the obtained results are matched properly with those that are reported by Magyari and Keller (1999), Ishak (2011), Mukhopadhyay (2013) and Mabood et al. (2017) which are given in Table 2 . That encourages us indicating the accuracy of present numerical results.

The numerical solutions of Eq. 8 and 9 subjecting to boundary conditions (Eq. 10) are obtained by applying the shooting technique. Dual solutions are found for $\mathrm{f}^{\prime \prime}(0)$ and $\theta^{\prime}(0)$ for the different applied parameters at the

Table 1: Thethrmophy sical properties of nanoparticles and water

\begin{tabular}{lccc}
\hline Fluids & $\rho\left(\mathrm{kg} / \mathrm{m}^{3}\right)$ & $\mathrm{C}_{p}(\mathrm{~J} / \mathrm{kg} \mathrm{K})$ & $\mathrm{k}(\mathrm{W} / \mathrm{mK})$ \\
\hline Silver $(\mathrm{Ag})$ & 10500 & 235 & 429 \\
Copper $(\mathrm{Cu})$ & 8933 & 385 & 400 \\
Aluminium & 2701 & 902 & 237 \\
Pure water & 997.1 & 4179 & 0.613 \\
\hline
\end{tabular}

different initial guesses. Furthermore, numerical calculations are performed to find the flow and the heat transfer profiles along with the solid nanoparticles. We have applied the field boundary conditions at the finite value the similarity variable $\eta$ that is denoted as $\eta_{\infty}$. The bulk computations are performed at minimum value $\eta_{\infty}=2$ and the maximum value $\eta_{\infty}=6$ that are the appropriate to obtain the field boundary conditions a symptotically at the values of considered parameters.

To clarify the salient features concerned to flow as well as heat transfer phenomena, the numerically obtained results have been presented in Fig. 1-17. Variations of the $\mathrm{f}^{\prime \prime}(0)$ and $\theta^{\prime}(0)$ along the suction parameter are presented in Fig. 2 and 3 for the few values of nanoparticles volume fraction $\phi$ for $\mathrm{Cu}$-water functioning fluid. Figure 2 and 3 represents the regions of no solutions for $\mathrm{S}<\mathrm{S}_{\mathrm{c} 1}, \mathrm{~S}<\mathrm{S}_{\mathrm{c} 2}$ and $\mathrm{S}<\mathrm{S}_{\mathrm{c} 3}$ and dual (first and second solution) solutions for $\mathrm{S}_{\mathrm{c} 1}<\mathrm{S}, \mathrm{S}_{\mathrm{c} 2}<\mathrm{S}$ and $\mathrm{S}_{\mathrm{c} 2}<\mathrm{S}$. When suction is increased, the skin friction is also increased in the first solution whereas the opposite trend is noticed in the second solution (Fig. 2). On the other hand, heat transfer rate increases as suction increases in both solutions. It is worth mentioning, here, that the exponential shrinking surface producesa higher rate of the shrinking as compare to the linear as well as non-linear shrinking surfaces in result an amount of the vorticity is generated because of exponential shrinking is larger than linear and non-linear shrinking. Bhattacharyya (2011) explained the dual

Table 2: The compression of values of heat transfer for various values of $P_{r}$

\begin{tabular}{|c|c|c|c|c|c|c|}
\hline$\overline{\mathrm{M}}$ & $\mathrm{P}_{Y}$ & Mabood et al. (2017) & Mukhopadhyay (2013) & Magyari and Keller (1999) & Ishak (2011) & Present results \\
\hline \multirow[t]{5}{*}{0} & 1 & 0.95478 & 0.9547 & 0.9548 & 0.9548 & 0.9548106 \\
\hline & 2 & 1.47151 & 1.4714 & -- & 1.4715 & 1.4714540 \\
\hline & 3 & 1.86909 & 1.8691 & 1.8691 & 1.8691 & 1.8690687 \\
\hline & 5 & 2.50012 & 2.5001 & 2.5001 & 2.5001 & 2.5001279 \\
\hline & 10 & 3.66039 & 3.6603 & 3.6604 & 3.6604 & 3.6603693 \\
\hline
\end{tabular}

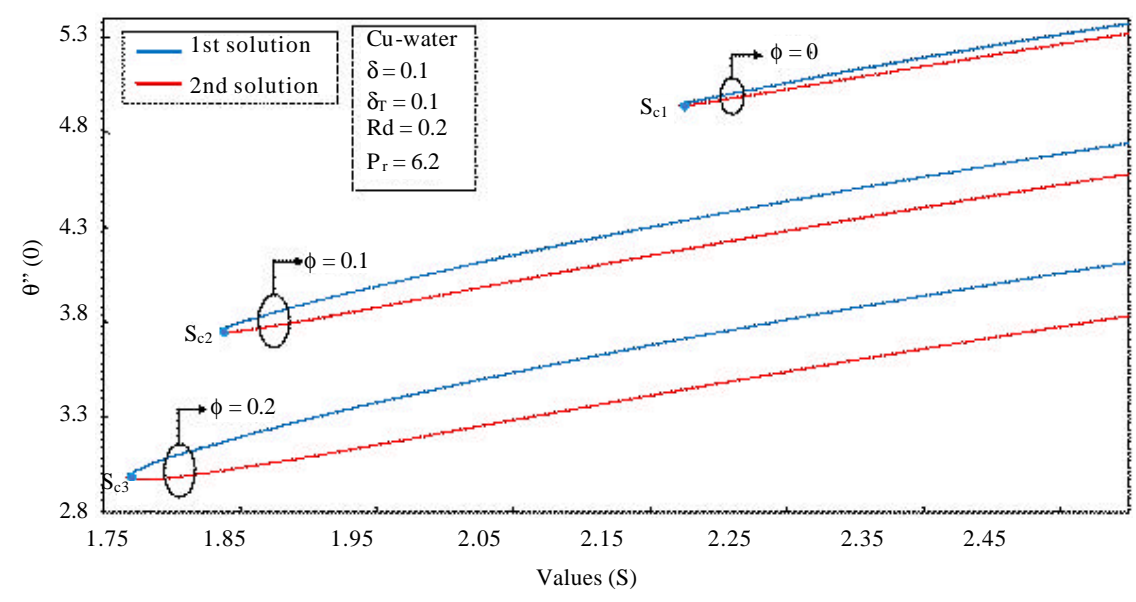

Fig. 2: The variation of $-\theta^{\prime}(0)$ along $\delta$ at different values of $\phi(0 \leq \phi \leq 0.2)$ for the Cu-water operational fluid 


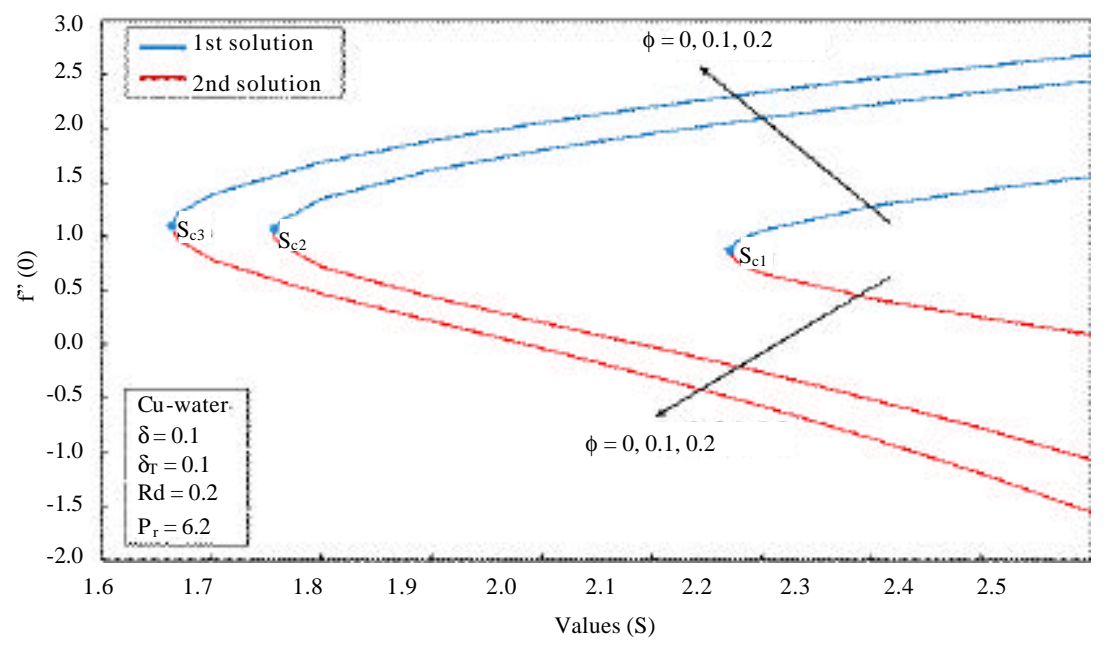

Fig. 3: The variation of $\mathrm{f}^{\prime \prime}(0)$ along $\delta$ at different values of $\phi(0 \leq \phi \leq 0.2)$ for the Ag-water operational fluid

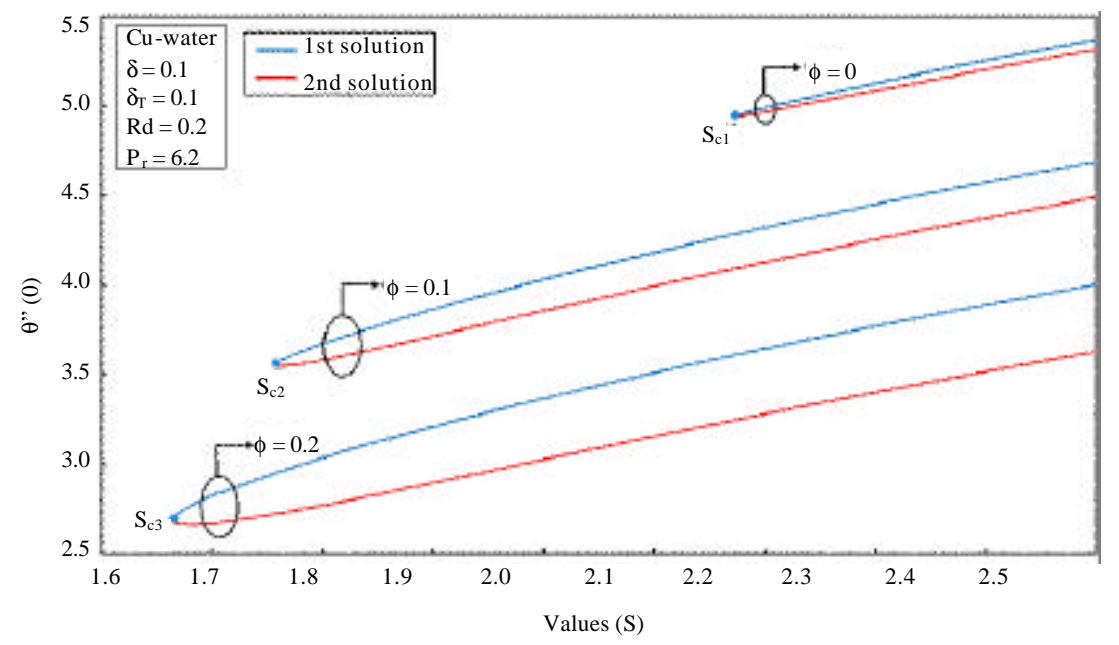

Fig. 4: The variation of $-\theta^{\prime}(0)$ along $\delta$ at different values of $\phi(0 \leq \phi \leq 0.2)$ for theAg-water operational fluid

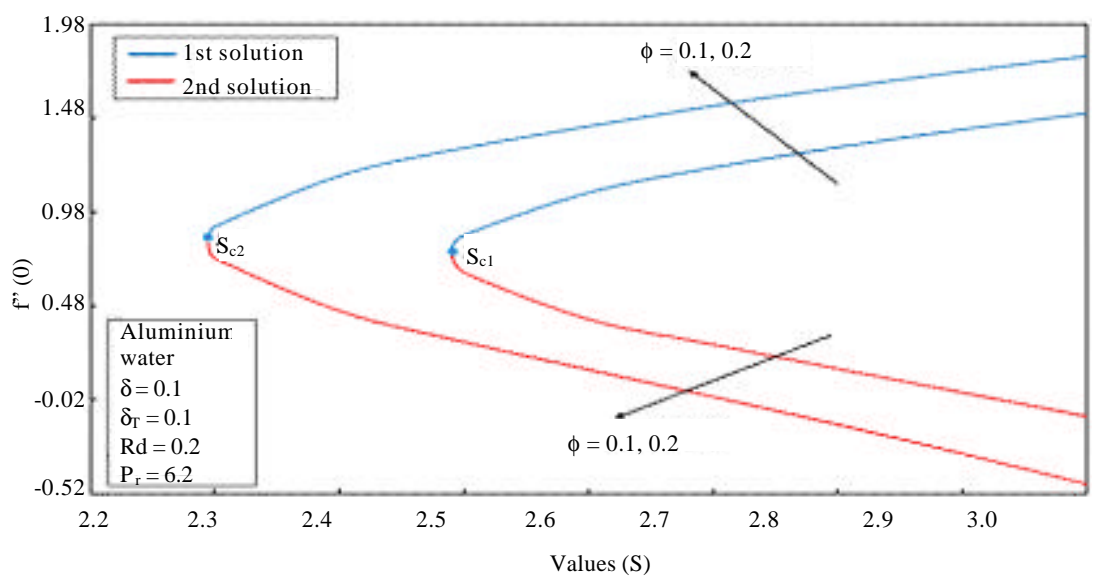

Fig. 5: The variation of $f^{\prime \prime}(0)$ along $\delta$ at different values of $\phi(0 \leq \phi \leq 0.2)$ for the aluminum-water operational fluid 


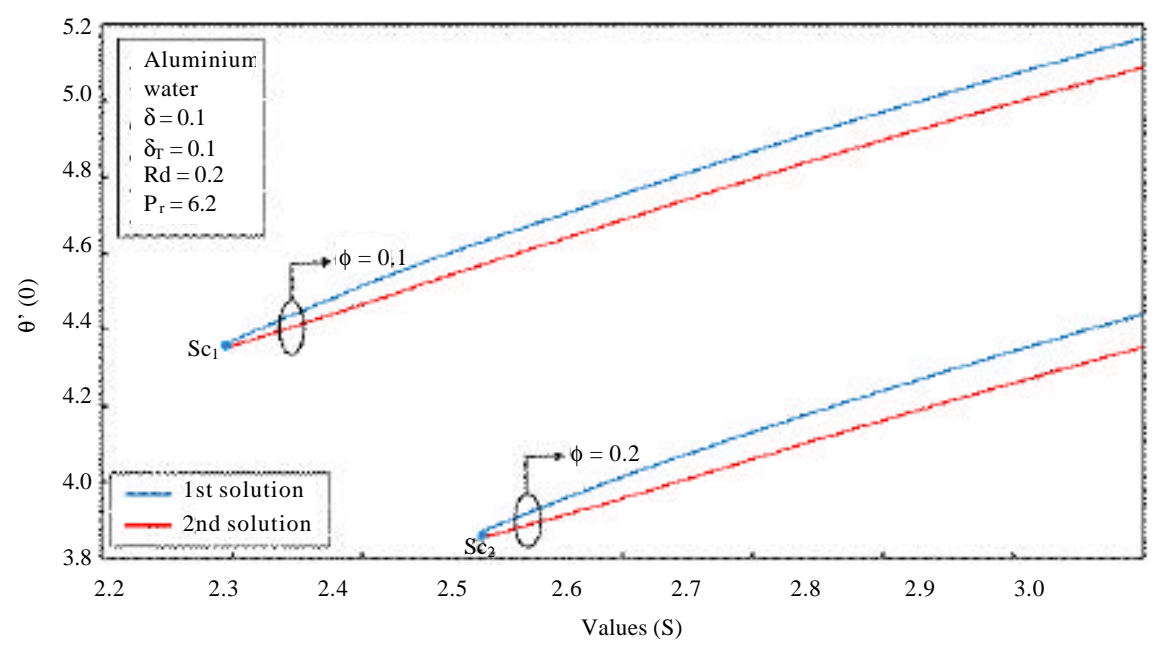

Fig. 6: The variation of $-\theta(0)$ along $\delta$ at different values of $\phi(0 \leq \phi \leq 0.2)$ for the aluminum-water operational fluid

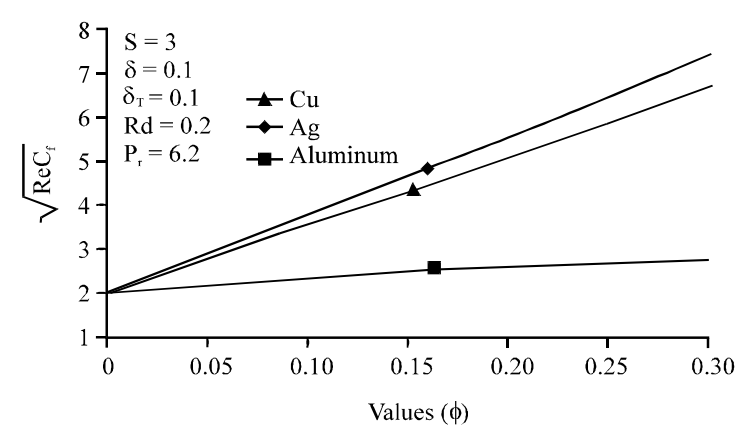

Fig. 7: The variation of skin friction coefficient along $\phi$ for the used specific nanoparticles

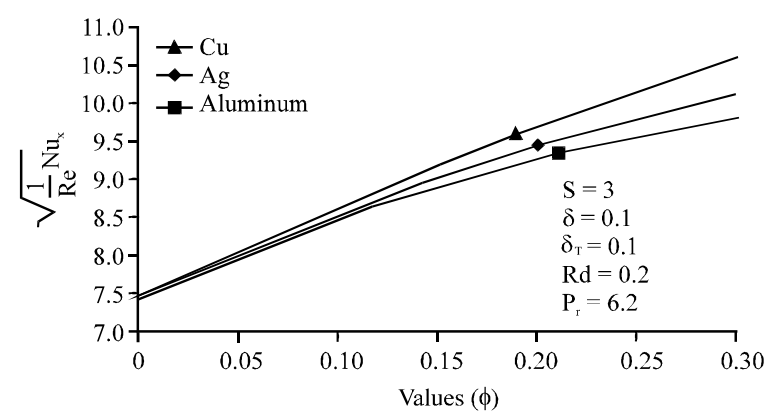

Fig. 8: The variation of thelocal Nusselt number along the $\phi$ for the used specific nanoparticles

similarity solutions in case of the newtonian fluid on the exponentially shrinking sheet may be determined at the value of suction parameter $\mathrm{S} \geq 2.266684$.

Hence, the larger value of the vorticity produced in boundary layer flow on the exponentially shrinking surfaces needs more suction as compared to flow over the non-linearly and linearly shrinking surfaces. From stability
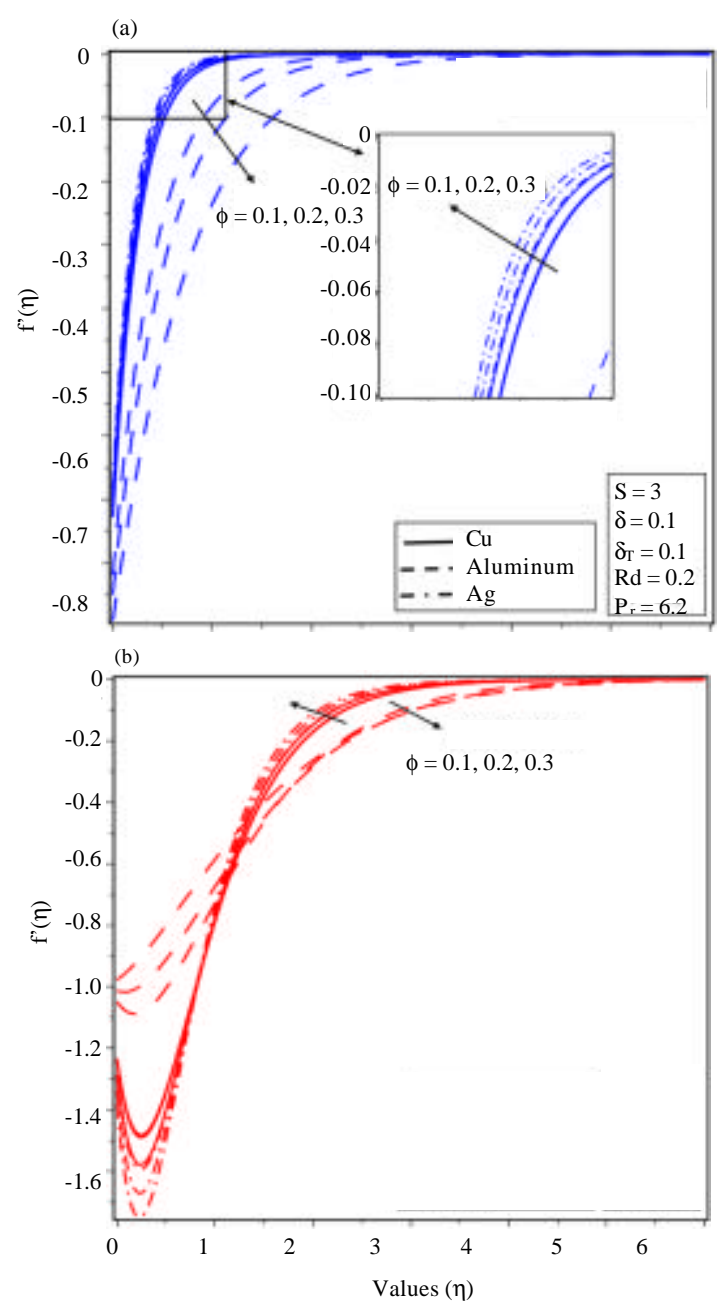

Fig. 9: Velocity profiles $f^{\prime}(\eta)$ vs. $\eta$ for different values of $\phi$ for different nanoparticles: a) 1 st solution and b) 2nd solution 
(a)

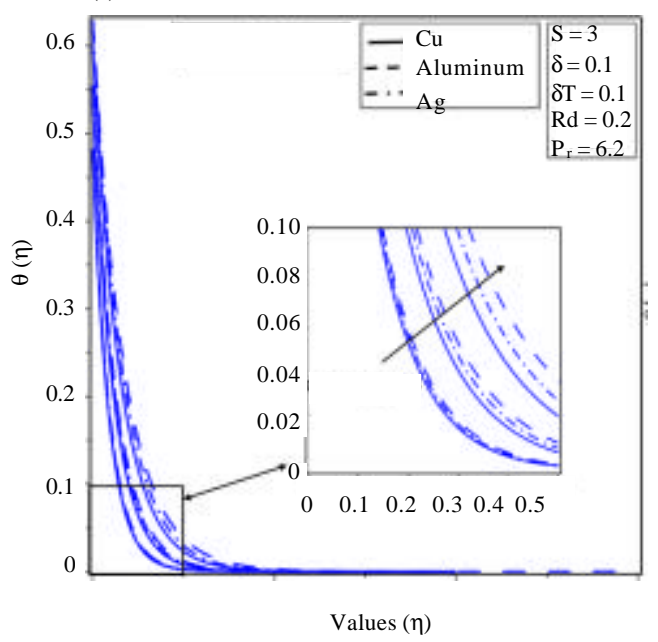

(b)

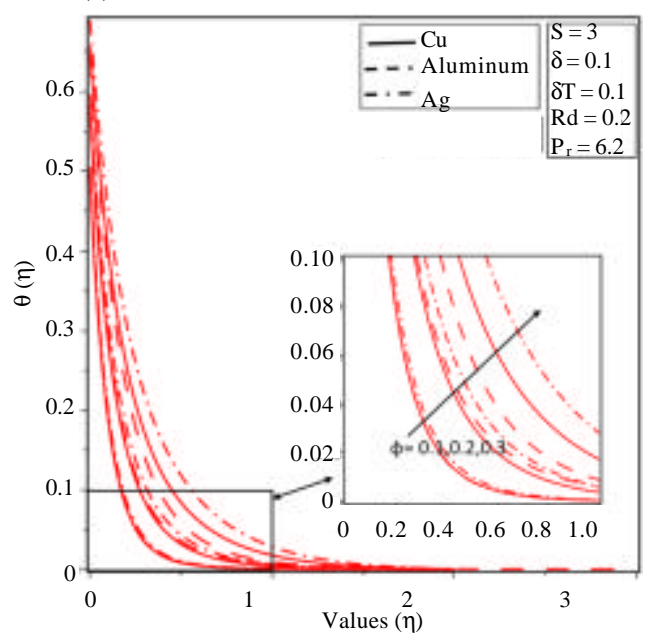

Fig. 10: Temperature profiles $\theta(\eta)$ vs. $\eta$ for different values of $\phi$ for different nanoparticles: a) 1 st solution and b) 2 nd solution

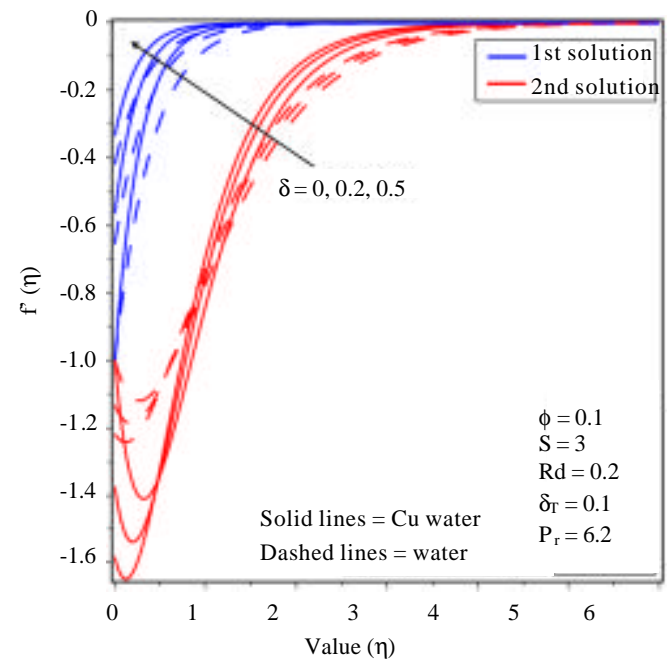

Fig. 11: Velocity profiles $\mathrm{f}^{\prime}(\eta)$ (Cu-water) vs. $\eta$ for different values of velocity $\operatorname{slip} \delta$

analysis, it is determined the first solution, represented the upper branch of graphs is stable and the physically meaningful while the second solutions represented in the lower branch of graphs are unstable and not acceptable. Table 3 and 4 represent smallest eigenvalues $\varepsilon$ of the first and second solutions for the numerous values of the velocity slip parameter $\delta$ and the thermal slip parameter $\boldsymbol{\delta}_{\mathrm{T}}$. The numerical results of wall skin friction coefficient $\mathrm{f}^{\prime}(0)$ as well as wall Nusselt number $\theta^{\prime}(0)$ of Ag-water based nanofluid at the wide range of suction parameter $\mathrm{S}$, are represented in Fig. 3 and 4, respectively. Figure 3 represents that at the different values of the $S$, values of the $f^{\prime \prime}(0)$ are positive that indicates the surface does not

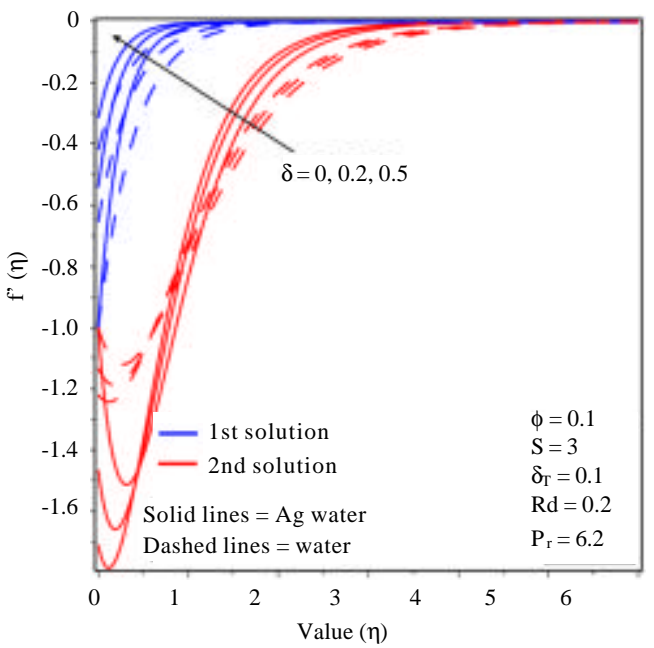

Fig. 12: The velocity profiles $f^{\prime}(\eta)$ (Ag-water) vs. $\eta$ at the different values of velocity slip $\delta$

Table 3: Value of turning points of all kinds of fluid

\begin{tabular}{llll}
\hline Turning points & $\mathrm{S}_{\mathrm{c} 1}$ & $\mathrm{~S}_{\mathrm{c} 2}$ & $\mathrm{~S}_{\mathrm{c} 3}$ \\
\hline $\mathrm{Cu}$ & 2.17154 & 1.83538 & 1.7671 \\
Aluminium & 2.29402 & 2.48986 & --- \\
$\mathrm{Ag}$ & 2.17154 & 1.75648 & 1.66405 \\
\hline
\end{tabular}

Table 4: Smallest eigen values of all kinds of fluid when $ø=0.1, \mathrm{Rd}=0.2$ and $P_{Y}=6.2$

\begin{tabular}{lllll}
\hline & & & & \\
Elements & $\delta$ & $\delta_{\mathrm{T}}$ & & \\
\hline $\mathrm{Cu}$ & 0.0 & 0.1 & 2.6601 & 1st solution \\
& 0.1 & 0.0 & 2.9005 & 2nd solution \\
\hline \multirow{2}{*}{$\mathrm{Ag}$} & 0.1 & 0.1 & 3.05132 & -2.0799 \\
& 0.0 & 0.1 & 3.0043 & -2.3951 \\
Aluminium & 0.1 & 0.1 & 2.7431 & -1.6076 \\
& 0.0 & 0.1 & 3.2591 & -1.7267 \\
& & & & -1.9284 \\
\hline
\end{tabular}


J. Eng. Applied Sci., 14 (13): 4569-4582, 2019

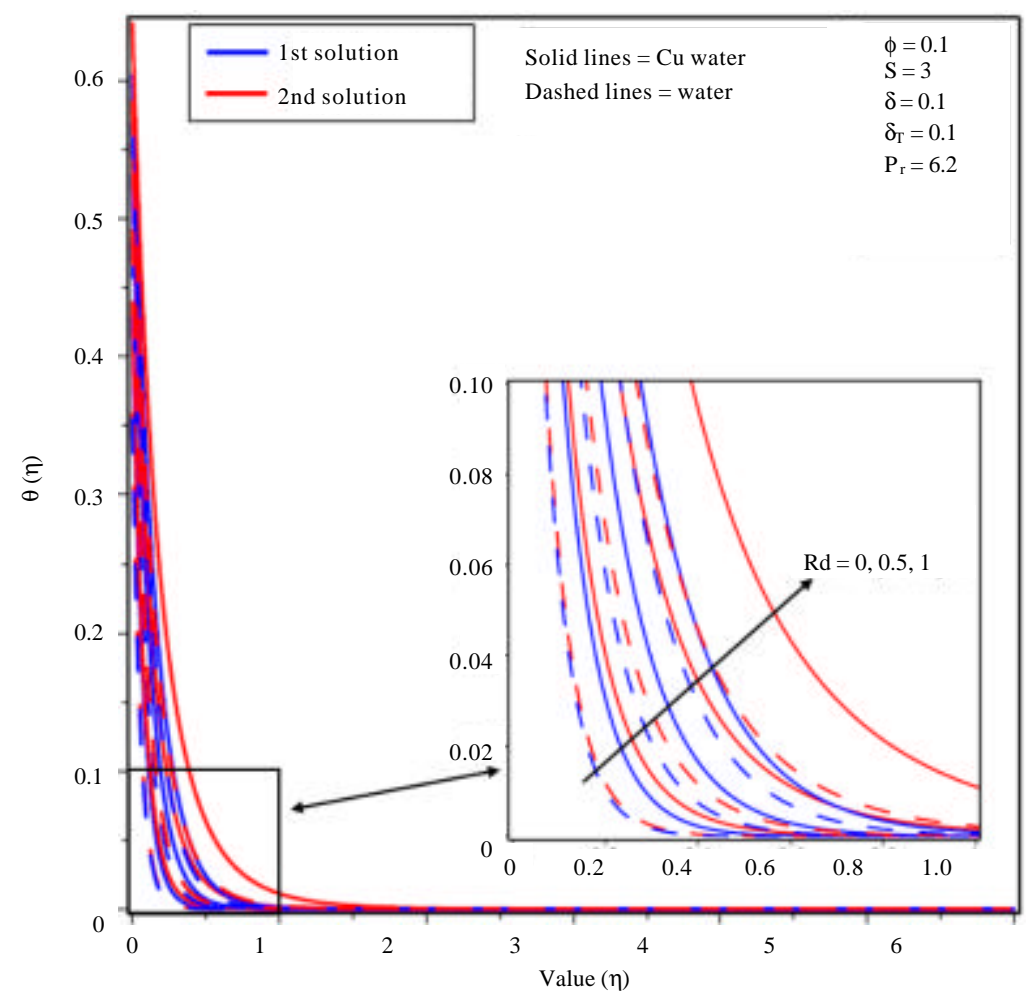

Fig. 13: The temperature profiles $\theta(\eta)$ (Cu-water) vs. $\eta$ at different values of thermal Radiation (Rd)

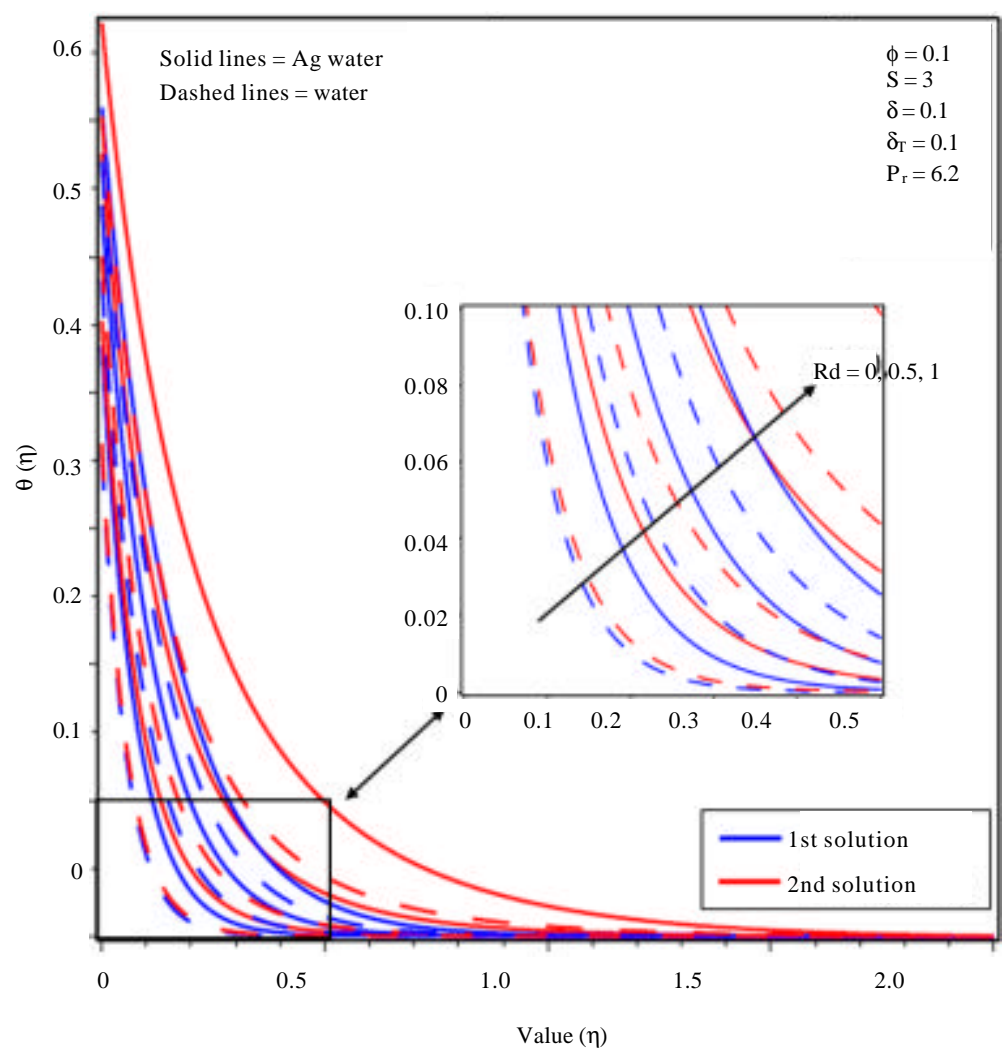

Fig. 14: The temperature profiles $\theta(\eta)$ (Ag-water) vs. $(\eta)$ at different values of thermal Radiation (Rd) 


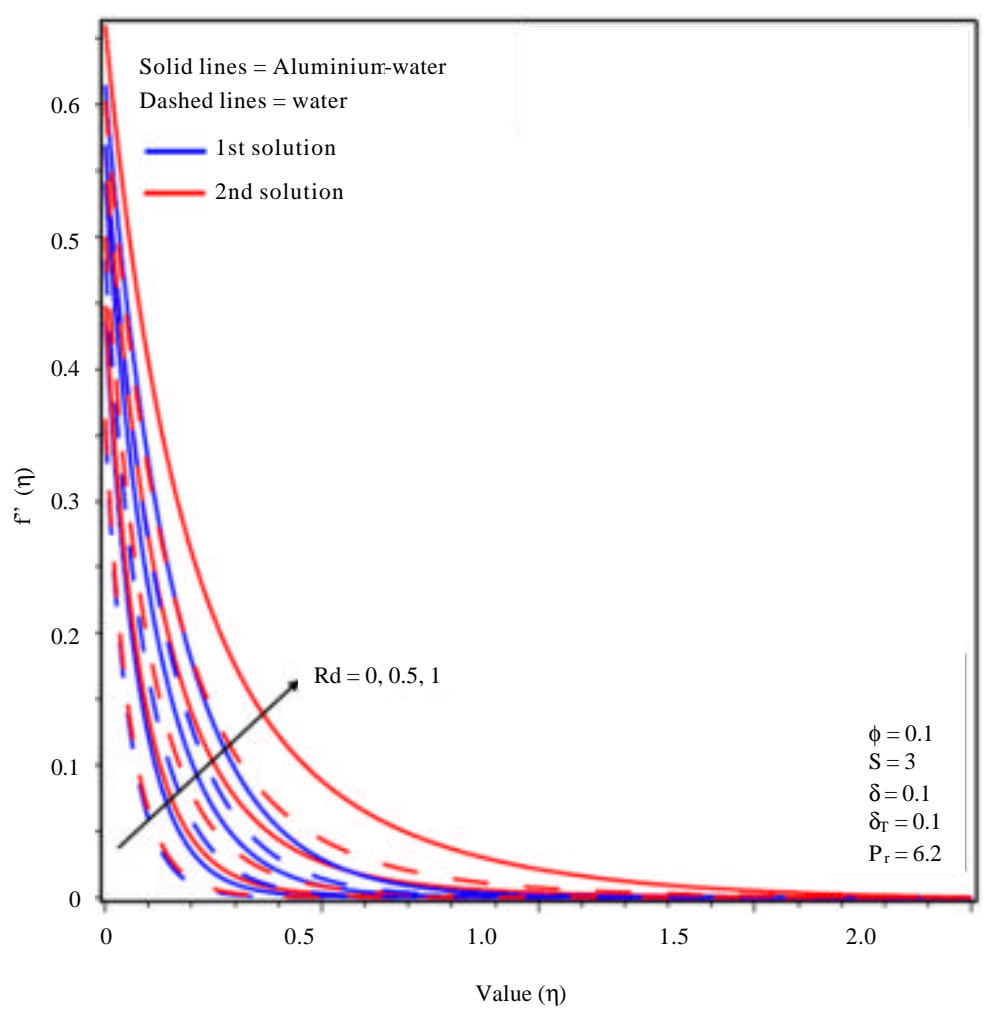

Fig. 15: The temperature profiles $\theta(\eta)$ (aluminium-water) vs. $\eta$ at different values of thermal Radiation (Rd)

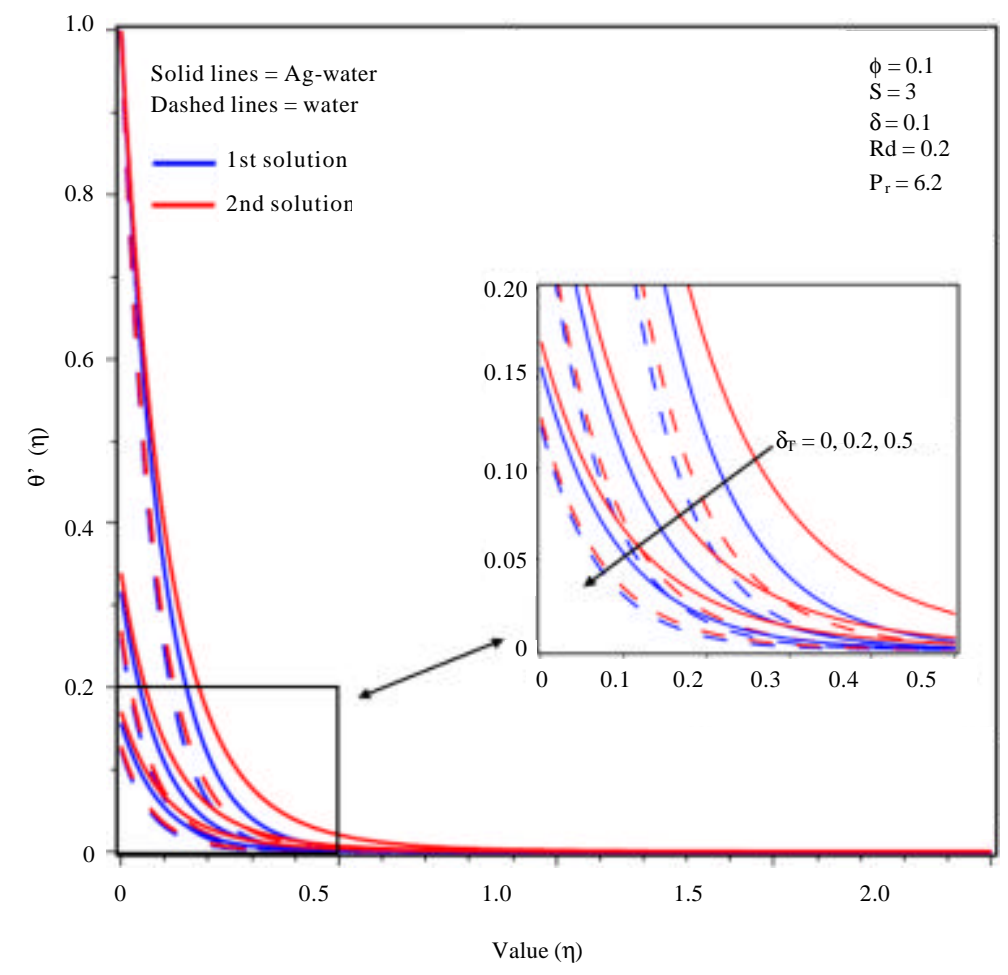

Fig. 16: The temperature profiles $\theta(\eta)$ (Ag-water) vs. $\eta$ at different values of thermal $\operatorname{slip}\left(\boldsymbol{\delta}_{\mathrm{T}}\right)$ 


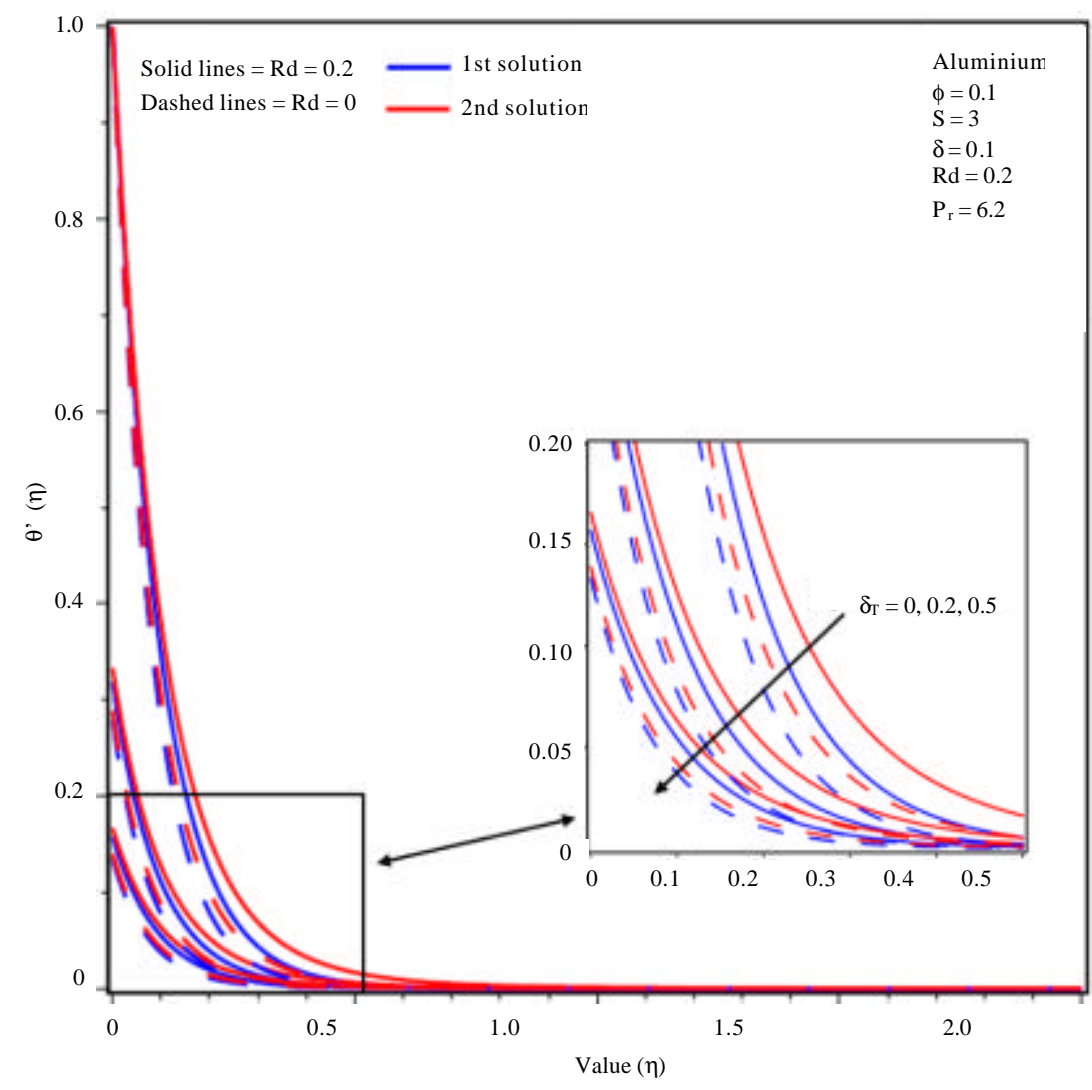

Fig. 17: The temperature profiles $\theta(\eta)$ (aluminium-water) vs. $\eta$ at different values of thermal slip $\left(\boldsymbol{\delta}_{\mathrm{T}}\right)$

exert the more drag force at the flowing fluid. It is observed that the skin friction at the surface is increased in the first solution while it is decreasing in the second solution as $\mathrm{S}$ is increased. On the other side, the heat transfer rate increases in both solutions when $\mathrm{S}$ is increased and the same pattern also has been noticed for aluminium-water based nanofluid (Fig. 6). Similarly, skin friction values of aluminium-water based nanofluid are despite in Fig. 5. It is examined that skin friction decreases in the second solution and increases in the first solution, when suction is increased. Figure 7 and 8 demonstrate variations of skin friction coefficient $\sqrt{\mathrm{Re}_{\mathrm{e}} \mathrm{C}_{\mathrm{f}}}$ and local Nusselt number $\sqrt{1 /(\mathrm{Re})} \mathrm{Nu}_{\mathrm{x}}$ presented in Eq. 12 along volume fraction nanoparticle parameter $\phi$ for the nanoparticles, Copper $(\mathrm{Cu})$, silver $(\mathrm{Ag})$ and aluminium. The figures represent that both quantities are increasing linearly by increasing rate of the $\phi$. The existence of solid nanoparticles in fluids increasing the thermal conductivity of fluids appreciably and therefore, enhancing the rate of heat transfer characteristics that can be seen in Fig. 8. The nanofluids possess the distinct characteristics that are different from the common liquid-solid mixtures where micrometer or millimeter-sized particles are used. Such particles can increase the pressure drop and can also clot equipment because of settling effects or settling down. Besides, they settled down rapidly due to more mass, so, the additional large pressure is created (Khanafer et al., 2003). Also, it is noticed, the least rate of heat transfer is observed in aluminium-water nanofluid because of the dominance of the conducting mode of the heat transfer. That is because of the aluminium possess the least thermal conductivity as compared to the $\mathrm{Cu}$ and $\mathrm{Ag}$ as given in Table 1. However, the variation in values of the $\mathrm{Cu}$-water and the Ag-water is not too large. The rate of the thermal conductivity in the case of $\mathrm{Ag}$ is greater than $\mathrm{Cu}$ as can be seen in Table 1, therefore, the largest rate of the heat transfer is observed in Ag-water nanofluid. On the other hand, it is detected that the skin friction growing by increasing solid fraction. Whereas silver-water nanofluid indicate faster growth than copper and aluminium water-based nanofluids. Figure 9 shows that momentum boundary layer thickness for Ag-water nanofluid is smaller than the rest of the nanofluids in both solutions, consequently, $\mathrm{Ag}$-water nanofluid tends to flow closer to the convectively shrinking surface and is the more coolant than the other nanofluids. Figure 10 
indicates that the temperature is maximum at the surface due to the conventional heating but it is decreasing to zero far away from the surface which satisfies free stream condition. It is observed from Fig. 10 that the surface temperature is highest for Ag-water nanofluid followed by $\mathrm{Cu}$-water nanofluid while aluminium-water nanofluid exhibited the least temperature at the shrinking surface in both solutions. The effects of velocity slip on velocity profile are represented in Fig. 11. It is examined that thickness of the hydrodynamic boundary layer is decreasing in both solution and both cases $(\phi=0.1$ and $\phi=0$ ) when slip $\delta$ is increased. Figure 12 shows the effect of velocity slip on the velocity profile of Ag-water nanofluid. Velocity profile shows the same behavior as, we already noticed in Fig. 11. That is because of the fact that with increasing $\delta$ the resistance of in fluid increases, in result velocity of the flowing fluid is decreased. Figure 13-15 indicate temperature distribution $\theta(\eta)$ with different values of thermal Radiation parameter (Rd) for $\mathrm{Cu}, \mathrm{Ag}$ and aluminium-water cases, respectively. It is noticed in both solutions of all temperature profiles of $\mathrm{Cu}$, $\mathrm{Ag}$ and aluminium-water nanofluid that thickness of thermal boundary layers increases as radiation parameter $\mathrm{Rd}$ is increased. Figure 16 shows the effect of slip parameter on the temperature profile of Ag-water nanofluid. From this, it is examined that the temperature decreases by increasing $\delta_{\mathrm{T}}$ in both solutions. When thermal slip parameter $\delta_{\mathrm{T}}$ is increased in a result temperature and thickness of the thermal boundary layer of aluminium-water nanofluid decreases (Fig. 17).

\section{CONCLUSION}

There has been studied the influence of the different solid nano-sized particle volumetric fractions at the two-dimensional boundary layer water-based nanofluid flow on the exponentially shrinking surface. The influence of the Copper $(\mathrm{Cu})$, silver $(\mathrm{Ag})$ and the aluminium volumetric fractions are considered by using Tiwari and Dass (2007) model. There has been examined the effects of applied parameters along with nanoparticles volumetric fractions, the velocity and the thermal slip parameters and also the radiation parameter on both velocity and temperature profiles. Besides it, the skin frictions and the Nussult number are considered. The main findings are given as:

- The velocity profilesare decreasedby increasing the nanoparticle volume fraction and velocity slip parameters in both solutions

- The temperature profiles also decrease in both solutions when a thermal slip parameter is increased
- The increase of suction parameter increases the rate of skin frictions in the first solution whereas opposite to it in the second solution it decreases

- Heat transfers increase in both solutions with the increase in the suction parameter

- At Ag-nanoparticles moresuspension in the water, the rate of resistance to flow becomes higher as compared to $\mathrm{Cu}$ and aluminium

- The increment in thermal radiation parameter increases the temperature profiles

\section{ACKNOWLEDGEMENT}

The researchers would like to acknowledge Universiti Utara Malaysia for financial support through University Grant (S/O Code: 13873).

\section{REFERENCES}

Aghamajidi, M., M. Yazdi, S. Dinarvand and I. Pop, 2018. Tiwari-Das nanofluid model for Magnetohydrodynamics (MHD) natural-convective flow of a nanofluid adjacent to a spinning down-pointing vertical cone. Propul. Power Res., 7: 78-90.

Ahmad, S., A.M. Rohni and I. Pop, 2011. Blasius and sakiadis problems in nanofluids. Acta Mechanica, 218: 195-204.

Bachok, N., A. Ishak and I. Pop, 2012. Boundary layer stagnation-point flow and heat transfer over an exponentially stretching/shrinking sheet in a nanofluid. Int. J. Heat Mass Transfer, 55: 8122-81 28.

Bhattacharyya, K. and K. Vajravelu, 2012. Stagnation-point flow and heat transfer over an exponentially shrinking sheet. Commun. Nonlinear Sci. Numer. Simul., 17: 2728-2734.

Bhattacharyya, K., 2011. Boundary layer flow and heat transfer over an exponentially shrinking sheet. Chin. Phys. Lett., 28: 074701-1-074701-4.

Buongiorno, J., 2006. Convective transport in nanofluids. ASME J. Heat Transfer, 128: 240-250.

Choi, S.U.S. and J.A. Eastman, 1995. Enhancing Thermal Conductivity of Fluids with Nanoparticles. In: Developments and Applications of Non-Newtonian Flows, Siginer, D.A. and H.P. Wang (Eds.). American Society of Mechanical Engineers, New York, USA., pp: 99-105.

Emami, R.Y., M. Siavashi and G.S. Moghaddam, 2018. The effect of inclination angle and hot wall configuration on $\mathrm{Cu}$-water nanofluid natural convection inside a porous square cavity. Adv. Powder Technol., 29: 519-536. 
Hamid, A. and M. Khan, 2018. Numerical simulation for heat transfer performance in unsteady flow of Williamson fluid driven by a wedge-geometry. Results Phys., 9: 479-485.

Hamid, A., M. Khan and A. Hafeez, 2018. Unsteady stagnation-point flow of Williamson fluid generated by stretching/shrinking sheet with Ohmic heating. Intl. J. Heat Mass Transfer, 126: 933-940.

Harris, S.D., D.B. Ingham and I. Pop, 2009. Mixed convection boundary-layer flow near the stagnation point on a vertical surface in a porous medium: Brinkman model with slip. Trans. Porous Media, 77: 267-285.

Hayat, T., M. Imtiaz and A. Alsaedi, 2016. Melting heat transfer in the $\mathrm{MHD}$ flow of $\mathrm{Cu}$-water nanofluid with viscous dissipation and Joule heating. Adv. Powder Technol., 27: 1301-1308.

Hayat, T., M. Rashid, A. Alsaedi and B. Ahmad, 2018b. Flow of nanofluid by nonlinear stretching velocity. Results Phys., 8: 1104-1109.

Hayat, T., S. Ayub, A. Tanveer and A. Alsaedi, 2018a. Numerical simulation for MHD Williamson fluid utilizing modified Darcys law. Results Phys., 10: 751-759.

Ishak, A., 2011. MHD boundary layer flow due to an exponentially stretching sheet with radiation effect. Sains Malaysiana, 40: 391-395.

Ismail, N.S., N.M. Arifin, N. Bachok and N. Mahiddin, 2016. Stagnation-point flow and heat transfer over an exponentially shrinking sheet: A stability analysis. AIP. Conf. Proc., Vol. 1739 ,

Kashyap, D. and A.K. Dass, 2018. Two-phase lattice Boltzmann simulation of natural convection in a $\mathrm{Cu}$-water nanofluid-filled porous cavity: Effects of thermal boundary conditions on heat transfer and entropy generation. Adv. Powder Technol., 29: 2707-2724.

Khan, U., N. Ahmed and S.T. Mohyud-Din, 2018. Analysis of magnetohydrodynamic flow and heat transfer of Cu-water nanofluid between parallel plates for different shapes of nanoparticles. Neural Comput. Appl., 29: 695-703.

Khan, W.A. and I. Pop, 2010. Boundary-layer flow of a nanofluid past a stretching sheet. Int. J. Heat Mass Transfer, 53: 2477-2483

Khanafer, K., K. Vafai and M. Lightstone, 2003. Buoyancy-driven heat transfer enhancement in a two-dimensional enclosure utilizing nanofluids. Int. J. Heat Mass Transfer, 46: 3639-3653.

Kumaran, G. and N. Sandeep, 2017. Thermophoresis and Brownian moment effects on parabolic flow of $\mathrm{MHD}$ Casson and Williamson fluids with cross diffusion. J. Mol. Liq., 233: 262-269.
Mabood, F., W.A. Khan and A.M. Ismail, 2017. MHD flow over exponential radiating stretching sheet using homotopy analysis method. J. King Saud Univ. Eng. Sci., 29: 68-74.

Magyari, E. and B. Keller, 1999. Heat and mass transfer in the boundary layers on an exponentially stretching continuous surface. J. Phys. D: Applied Phys., 32: 557-585.

Masuda, H., A. Ebata, K. Teramae and N. Hishinuma, 1993. Alteration of thermal conductivity and viscosity of liquid by dispersing ultra fine particles. NetsuBussei, 7: 227-233.

Maxwell, J.C., 1879. On stresses in rarified gases arising from inequalities of temperature. Philos. Trans. R. Soc. London, 170: 231-256.

Mukhopadhyay, S., 2013. Casson fluid flow and heat transfer over a nonlinearly stretching surface. Chin. Phys. B., 22: 074701-1-074701-5.

Mustafa, M., J.A. Khan, T. Hayat and A. Alsaedi, 2018. Numerical solutions for radiative heat transfer in ferrofluid flow due to a rotating disk: Tiwari and Das Model. Intl. J. Nonlinear Sci. Numer. Simul., 19: 1-10.

Nield, D.A. and A.V. Kuznetsov, 2009. The Cheng-Minkowycz problem for natural convective boundary-layer flow in a porous medium saturated by a nanofluid. Int. J. Heat Mass Transfer, 52: 5792-5795.

Nield, D.A. and A.V. Kuznetsov, 2011. The Cheng-Minkowycz problem for the double-diffusive natural convective boundary layer flow in a porous medium saturated by a nanofluid. Int. J. Heat Mass Transfer, 54: 374-378.

Ramzan, M., M. Bilal and J.D. Chung, 2017. MHD stagnation point Cattaneo-Christov heat flux in Williamson fluid flow with homogeneousheterogeneous reactions and convective boundary condition-a numerical approach. J. Mol. Liq., 225: 856-862.

Rashad, A.M., M.M. Rashidi, G. Lorenzini, S.E. Ahmed and A.M. Aly, 2017. Magnetic field and internal heat generation effects on the free convection in a rectangular cavity filled with a porous medium saturated with $\mathrm{Cu}$-water nanofluid. Intl. J. Heat Mass Transfer, 104: 878-889.

Raza, J., A.M. Rohni and Z. Omar, 2016. MHD flow and heat transfer of $\mathrm{Cu}$-water nanofluid in a semi porous channel with stretching walls. Intl. J. Heat Mass Transfer, 103: 336-340.

Reddy, P.S., P. Sreedevi and A.J. Chamkha, 2017. MHD boundary layer flow, heat and mass transfer analysis over a rotating disk through porous medium saturated by $\mathrm{Cu}$-water and $\mathrm{Ag}$-water nanofluid with chemical reaction. Powder Technol., 307: 46-55. 
Rosca, A.V. and I. Pop, 2013. Flow and heat transfer over a vertical permeable stretching/shrinking sheet with a second order slip. Intl. J. Heat Mass Transfer, 60: 355-364.

Sandeep, N. and M.G. Reddy, 2017. Heat transfer of nonlinear radiative magnetohydrodynamic $\mathrm{Cu}$-water nanofluid flow over two different geometries. J. Mol. Liq., 225: 87-94.

Sheremet, M.A., I. Pop and N. Bachok, 2016. Effect of thermal dispersion on transient natural convection in a wavy-walled porous cavity filled with a nanofluid: Tiwari and Das nanofluid model. Intl. J. Heat Mass Transfer, 92: 1053-1060.

Shu, J.J., J.B.M. Teo and W.K. Chan, 2016. A new model for fluid velocity slip on a solid surface. Soft Matter, 12: 8388-8397.
Sulochana, C. and N. Sandeep, 2016. Stagnation point flow and heat transfer behavior of Cu-water nanofluid towards horizontal and exponentially stretching/shrinking cylinders. Appl. Nanosci., 6: 451-459.

Tiwari, R.K. and M.K. Das, 2007. Heat transfer augmentation in a two-sided lid-driven differentially heated square cavity utilizing nanofluids. Int. J. Heat Mass Transfer, 50: 2002-2018.

Weidman, P.D., D.G. Kubitschek and A.M.J. Davis, 2006. The effect of transpiration on self-similar boundary layer flow over moving surfaces. Int. J. Eng. Sci., 44: 730-737.

Xuan, Y. and W. Roetzel, 2000. Conceptions for heat transfer correlation of nanofluids. Int. J. Heat Mass Transfer, 43: 3701-3707. 